\title{
Suicídio em adultos jovens brasileiros: série temporal de 1997 a 2019
}

\author{
Suicide in young Brazilian adults: 1997-2019 time series
}

Vilmeyze Larissa de Arruda (https://orcid.org/0000-0003-1105-3880) ${ }^{1}$

Bruna Hinnah Borges Martins de Freitas (https://orcid.org/0000-0002-6652-593X) ${ }^{1}$

Samira Reschetti Marcon (https://orcid.org/0000-0002-5191-3331) ${ }^{1}$

Fabiana Yanes Fernandes (https://orcid.org/0000-0002-6367-5289) ${ }^{1}$

Nathalie Vilma Pollo de Lima (https://orcid.org/0000-0001-5558-972X) ${ }^{1}$

Juliano Bortolini (https://orcid.org/0000-0003-0126-3040) ${ }^{2}$

${ }^{1}$ Faculdade de Enfermagem, Universidade Federal de Mato Grosso (UFMT). Av. Fernando Corrêa da Costa 2367, Bairro Boa Esperança. 78060-900 Cuiabá MT Brasil. brunahinnah@hotmail.com ${ }^{2}$ Departamento de Estatística, UFMT. Cuiabá MT Brasil.

\begin{abstract}
This study aimed to analyze the time series of suicide mortality in Brazilian young adults between 1997 and 2019. This is an ecological study on time series with individuals aged 20 to 29 years. The Prais-Winsten regression was used for trend analysis. The mean suicide coefficient in young Brazilians was 6.36/100 thousand inhabitants in the period, showing increasing overall, females, and male trends. Regarding marital status, there was a higher proportion of suicide among single people (79.02\%). As for the Brazilian regions, the South presented the highest mean mortality coefficient (9.18/100 thousand inhabitants), and the Northeast, North, and Southeast regions showed an increasing trend. Most deaths occured at home (54.93\%), and there was an increasing trend both at home, at health establishments, and other places. We conclude that the suicide trend in Brazilian young adults was increasing in the period, with more significant proportions among single people. Brazilian regions showed different trends, and households, health facilities, and other locations followed an increasing trend toward suicide deaths in young adults.
\end{abstract}

Key words Suicide, Time series studies, Young adult
Resumo Este estudo teve como objetivo analisar a série temporal da mortalidade por suicídio em adultos jovens brasileiros entre os anos de $1997 e$ 2019. Trata-se de um estudo ecológico, de séries temporais com indivíduos de 20 a 29 anos. Para a análise de tendência foi utilizada a regressão de Prais-Winsten. No período, o coeficiente médio de suicídio em jovens brasileiros foi de 6,36/100 mil habitantes, apresentando tendência crescente, tanto o geral, quanto no sexo feminino e masculino. Em relação ao estado civil, houve maior proporção de suicídio entre solteiros $(79,02 \%)$. Quanto as regiões brasileiras, a Sul apresentou o maior coeficiente médio de mortalidade $(9,18 / 100$ mil habitantes), e as regiões Nordeste, Norte e Sudeste mostraram tendência crescente. A maioria dos óbitos ocorreram no domicílio (54,93\%) $e$ houve tendência crescente tanto no domicílio, como os estabelecimentos de saúde e outros locais. Conclui-se que a tendência do suicídio em adultos jovens brasileiros foi crescente no periodo, com maiores proporções entre os solteiros. As regiões brasileiras apresentaram diferentes tendências, e o domicílio, estabelecimentos de saúde e outros locais seguiram uma tendência crescente para as mortes por suicídio em adultos jovens.

Palavras-chave Suicídio, Estudos de séries temporais, Adulto jovem 


\section{Introdução}

O suicídio é compreendido como toda lesão autoprovocada, cuja intenção, mesmo que de forma ambivalente, seja a morte ${ }^{1}$. Configura-se como um fenômeno complexo e multifatorial que afeta familiares, comunidades e países, gerando impactos desestruturantes sobre as pessoas que faziam parte dos vínculos sociais das vítimas².

Em 2016, a taxa mundial de suicídio foi de $10,5 / 100$ mil habitantes, tornando-se a $15^{\mathrm{a}}$ causa de morte, e a segunda principal entre jovens de 15 a 29 anos. Quase 800.000 pessoas cometem o suicídio todos os anos, equivalendo a uma pessoa a cada 40 segundos ${ }^{2}$. Em termos globais, os suicídios matam mais que os homicídios e as guerras juntos ${ }^{3}$, e cerca de $79 \%$ dos casos ocorrem em países de baixa e média renda ${ }^{2}$.

No mesmo ano, o Brasil apresentou uma taxa geral de 6,5/100 mil habitantes, sendo que, no sexo masculino foi de 10,0/100 mil habitantes e o feminino 3,1/100 mil habitantes ${ }^{2}$. Essa diferença entre os sexos pode ser decorrente da maior propensão dos homens ao abuso de álcool, a escolhas de métodos mais letais na tentativa, maior inclinação para violência e comportamentos externalizantes, em comparação com as mulheres ${ }^{4}$.

Nos anos 2000, a mortalidade por suicídio diminuiu com os maiores declínios observados em alguns países da Europa Oriental. As tendências de queda foram seguidas por um aumento concomitante com a crise global de 2008 em alguns países, incluindo Grécia, Holanda e o Reino Unido, enquanto as tendências de queda antes da crise se mantiveram na Alemanha, Itália e Espanha. A análise também revelou ascensão no Brasil, México, Estados Unidos e Austrália ${ }^{5}$.

Em uma pesquisa desenvolvida no Brasil, observou-se aumento progressivo dos óbitos por suicídio na população geral em todas as regiões entre os anos de 1996 a $2015^{6}$. Entre as regiões brasileiras, constatou-se uma tendência crescente do suicídio na Norte, Nordeste e Sudeste. A diferença entre as demais pode ocorrer em consequência das diferentes características e condições socioeconômicas, culturais e ambientais. Contudo, em todas as regiões, a faixa etária com maior proporção de óbitos por suicídio foi a de 20 a 29 $\operatorname{anos}^{6}$.

Se constitui em um agravo de notificação compulsória, entretanto os dados relacionados a esse ato não são totalmente confiáveis, em decorrência das subnotificações ${ }^{7}$ suscitando, portanto, que esses indicadores sejam ainda maiores do que os descritos. A crise financeira global, os desastres naturais, a poluição do ar e fumo passivo estão associados ao aumento das taxas de suicídio. No nível individual, destacam-se o histórico de tentativa de suicídio, depressão, intoxicação por substâncias, a perda ou separação dos pais e outros eventos estressantes na vida ${ }^{8}$.

Destaca-se o domicílio como principal ambiente de escolha para o ato suicida devido à facilidade de acesso aos meios necessários para consumação do ato, como substâncias tóxicas, medicamentos, venenos como "chumbinho" e o enforcamento ${ }^{9,10}$. Outro local de grande ocorrência do evento são os hospitais ${ }^{9,10}$. A morte por suicídio pode ser causada por diferentes métodos, e as estatísticas demonstram que o enforcamento, a intoxicação exógena e as armas de fogo são predominantes na população mundial ${ }^{2,11,12}$.

Visto como um sério problema de saúde pública e de prevenção primordial e, considerando que apenas 28 países possuem estratégia nacional de prevenção, a Organização Mundial da Saúde (OMS) estabeleceu como meta a redução da taxa de suicídio mundial ${ }^{2}$. Contudo, são necessárias informações de qualidade para compreender $\mathrm{o}$ fenômeno e, assim, obter subsídios para a elaboração de políticas de prevenção ${ }^{7}$. Nesse sentido, este estudo tem como objetivo analisar a série temporal da mortalidade por suicídio em adultos jovens brasileiros entre os anos de 1997 e 2019.

\section{Método}

\section{Desenho de Estudo}

Trata-se de um estudo ecológico de séries temporais. A série temporal é uma maneira de organizar dados quantitativos de acordo com o tempo, possibilitando antever a distribuição de doenças na população, bem como fatores capazes de modificar essa distribuição ${ }^{13}$.

\section{Participantes}

Considerou-se como participantes do estudo a população de 20 a 29 anos que tiveram como causa básica de óbito o suicídio (códigos X60-X84), conforme a Classificação Estatística Internacional de Doenças e Problemas Relacionados com a Saúde (CID-10). A informação foi proveniente das estatísticas oficiais de mortalidade do Sistema de Informação sobre Mortalidade do Ministério da Saúde (SIM/MS). 


\section{Procedimento de Coleta de Dados}

Os dados foram coletados no Sistema de Informações sobre Mortalidade do Ministério da Saúde (SIM/MS) na página do Departamento de Informática do Sistema Único de Saúde (DATASUS). As estimativas populacionais por sexo, faixa etária (20 a 29 anos) e regiões foram obtidas do Instituto Brasileiro de Geografia e Estatística (IBGE).

O estudo foi realizado com dados do Brasil, maior país da América do Sul e quinto maior do planeta terra. É subdivido em cinco regiões (Norte, Nordeste, Sudeste, Sul e Centro-Oeste) e possui 27 Unidades de Federação.

O período de estudo foi de 1997 a 2019, totalizando 23 pontos, ou seja, 23 anos. Para analisar séries não é recomendada a utilização de menos de sete pontos, pois as tendências tendem a serem não significantes, devido ao pequeno poder estatístico da análise de regressão ${ }^{13}$.

\section{Variáveis}

A variável estudada foi o coeficiente de mortalidade específico por suicídio (X60-84). Além disso, foram analisadas as variáveis localização de residência (Brasil, regiões e estado), sexo (feminino e masculino), grupo de causas (lesão autoprovocada intencionalmente/X70-X84 e autointoxicação/X60-X69), estado civil (solteiro, casado, viúvo, separado judicialmente e outro) e local de ocorrência do óbito (hospital, outro estabelecimento e saúde, domicílio, via pública e outros). Entre os locais de ocorrência identificados como "outro estabelecimento de saúde", consideram-se os que não compreendem o serviço hospitalar, e entre os locais de ocorrência classificados como "outros", aqueles que não se enquadram em qualquer tipo de estabelecimento de saúde, domicílio ou via pública.

\section{Análise de dados}

Para o cálculo do coeficiente de mortalidade bruta específica por suicídio, o número de óbitos de residentes por suicídio (X60-84) foi considerado numerador e a população total residente ajustada ao meio do ano, denominador, multiplicado por $100 \mathrm{mil}^{14}$. Os coeficientes de mortalidade foram calculados por suicídio geral e segundo sexo, região e UF e, a mortalidade proporcional para as demais variáveis.

Para calcular a variação percentual anual (VPA) dos coeficientes, na análise de tendência, foi utilizada a regressão de Prais-Winsten, conforme descrita por Antunes e Cardoso ${ }^{13}$. Este modelo prevê a correção da autocorrelação de primeira ordem. A variável dependente foi o logaritmo dos coeficientes, e a variável independente, os anos da série histórica. A estimação quantitativa da tendência foi calculada pela seguinte expressão: $\mathrm{VPA}=\left[-1+10^{b}\right]{ }^{\star} 100 \%$. Para o cálculo dos intervalos de confiança (IC): IC95\%=[- $1+$ $10^{b \pm t^{*} s e}$.] ${ }^{\star} 100 \%$. Em que " $b$ " corresponde à taxa de crescimento anual. Os valores de " $b$ " e erro padrão (se) foram extraídos da análise de regressão, e o valor de " $t$ " será fornecido pela tabela da distribuição de $t$ de Student. A série foi considerada crescente quando a taxa foi positiva, decrescente quando negativa e estacionária quando não houve diferença significante entre seu valor e o zero $(\mathrm{p}>0,05)^{13}$. Esta análise foi realizada por meio do software STATA 11.1.

\section{Questões éticas}

Este estudo obteve aprovação do Comitê de Ética em Pesquisa (CEP) Saúde da Universidade Federal de Mato Grosso.

\section{Resultados}

Entre os anos de 1997 e 2019, foram registrados 48.076 óbitos por suicídio em jovens brasileiros. No período, o coeficiente médio bruto geral foi de 6,36 por 100 mil habitantes, 2,34 por 100 mil habitantes no sexo feminino e 10,39 por 100 mil habitantes no sexo masculino. Verificase na Figura 1 a tendência do coeficiente de mortalidade por suicídio em adultos jovens brasileiros, a qual expressou tendência crescente do coeficiente, tanto o geral (VPA: 1,26; IC95\%: 0,60;1,93), quanto o feminino (VPA: 1,45; IC95\%: 0,60;2,32) e masculino (VPA: 1,00; IC95\%: 0,57;1,43).

Quanto a tendência da mortalidade proporcional por grupo de causas relacionado ao suicídio, $87,18 \%(\mathrm{n}=41.913)$ dos óbitos no período relacionaram-se ao grupo de lesão autoprovocada (X70-X84), expressando tendência estacionária (VPA: 0,09; IC95\%: -0,18; 0,36) e 12,82\% $(n=6.163)$ corresponderam ao grupo de autointoxicação (X60-X69), com tendência decrescente (VPA: -1,79; IC95\%: -3,30; -0,24).

Entre as regiões do país, a Sul foi a que apresentou o maior coeficiente médio de mortalidade por suicídio (9,18/100 mil habitantes), mantendo tendência estável ao longo do período analisa- 


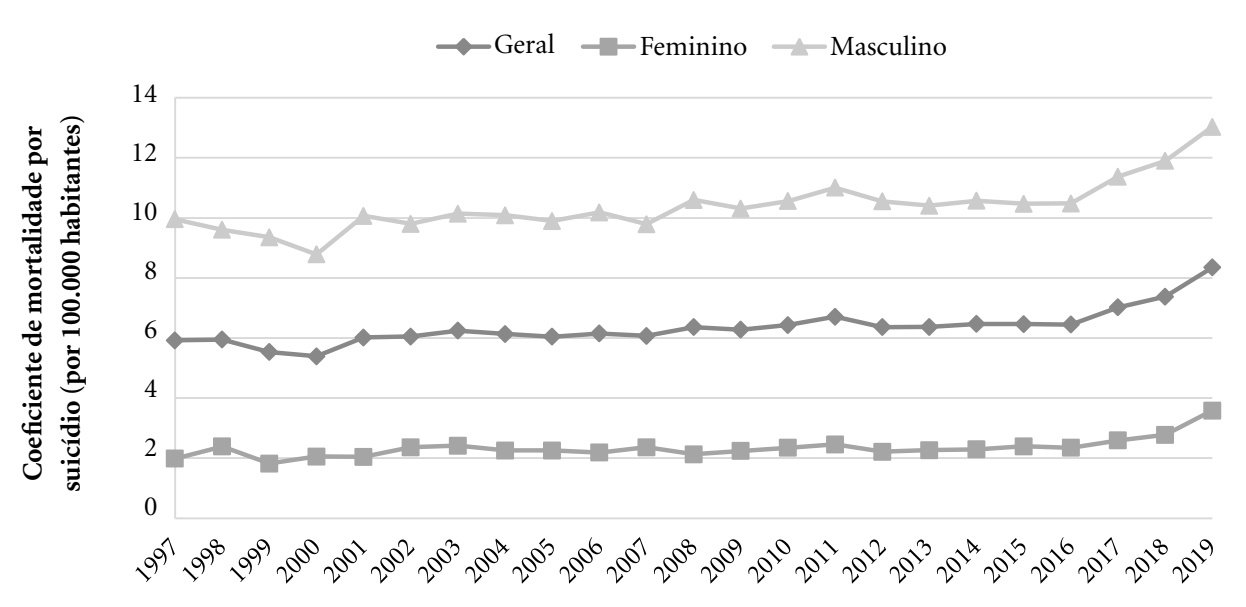

Figura 1. Tendência do coeficiente de mortalidade por suicídio em adultos jovens brasileiros, geral e por sexo. Brasil, 1997 a 2019.

Fonte: Sistema de Informação de Mortalidade (SIM), Ministério da Saúde (MS), 1997 a 2019.

do. Constata-se tendência de crescimento do coeficiente de mortalidade por suicídio em adultos jovens brasileiros no Nordeste, Norte e Sudeste, conforme pode ser visualizado na Tabela 1 .

No país, as três Unidades de Federação (UF) que apresentaram os maiores coeficiente de mortalidade por suicídio foram: Roraima $(13,54 / 100$ mil habitantes); Mato Grosso do Sul (11,40/100 mil habitantes) e Rio Grande do Sul (10,30/100 mil habitantes). Observa-se que, entre as $27 \mathrm{UF}$, a tendência foi crescente no Acre, Amazonas, Bahia, Maranhão, Mato Grosso do Sul, Minas Gerais, Pará, Paraíba, Piauí, Sergipe e Tocantins, segundo a Tabela 1. Apenas o Rio Grande do Sul apresentou tendência decrescente dos óbitos.

O domicílio foi o local de maior ocorrência do evento ( $n=26.406 ; 54,93 \%)$, seguido pelo hospital $(n=9.115 ; 18,96 \%)$. Destaca-se a tendência crescente do suicídio no domicílio, nos estabelecimentos de saúde e em outros locais (Tabela 2).

Em relação ao estado civil, houve maior proporção de suicídio entre solteiros $(n=37.988$; $79,02 \%)$. A tendência mostrou-se decrescente entre os casados e estacionária nos demais estados civis (Tabela 3).

\section{Discussão}

A tendência temporal do coeficiente de mortalidade bruta por suicídio em adultos jovens bra- sileiros no período de 1997 a 2019 foi crescente, tanto no sexo masculino, quanto no feminino. Constatou-se que, a tendência temporal das taxas estudadas mostrou diferentes padrões de acordo regiões, UF, locais de ocorrência do evento e estado civil. As variáveis que apresentaram tendência de aumento no período foram: as regiões Norte, Nordeste e Sudeste; as UF Acre, Amazonas, Bahia, Maranhão, Mato Grosso do Sul, Minas Gerais, Pará, Paraíba, Piauí, Sergipe e Tocantins; os óbitos ocorridos no domicílio, em outros estabelecimentos de saúde e em outros locais.

Pesquisas recentes desenvolvidas em outros países também apontam para um aumento acentuado da taxa de suicídio entre os mais jovens, como no Japão ${ }^{15}$, Estados Unidos ${ }^{16}$, Portugal $^{17}$ e Equador ${ }^{18}$. Esta é uma fase produtiva da vida e propícia a ocorrerem eventos significativos, como definições sobre vocação profissional, saída da casa dos pais, possível envolvimento amoroso, o ingresso em um curso superior, assim como o acesso mais fácil à substâncias psicoativas, que a depender da capacidade de enfrentamento do indivíduo, podem desencadear sofrimento psíqui$\mathrm{CO}^{19,20}$ predispondo esses jovens ao suicídio. Além disso, a crise econômica tem afetado milhares de jovens trabalhadores em diversos países e parece explicar esse aumento nas taxas de suicídio ${ }^{8,15}$.

Vale ressaltar que esses números provavelmente estão subestimados, considerando as subnotificações nos países de baixa e média renda 
Tabela 1. Número e porcentagem de óbitos, coeficiente médio por 100.000 habitantes e tendência dos coeficientes de mortalidade por suicídio, segundo Regiões e Unidade de Federação. Brasil, 1997-2019.

\begin{tabular}{|c|c|c|c|c|c|c|}
\hline \multirow{2}{*}{$\begin{array}{c}\text { Regiões e Unidade de } \\
\text { Federação }\end{array}$} & \multicolumn{2}{|c|}{ Óbitos } & \multirow{2}{*}{$\begin{array}{l}\text { Coeficiente } \\
\text { médio }\end{array}$} & \multirow{2}{*}{ VPA $^{a}$} & \multirow{2}{*}{ IC95\% ${ }^{\mathrm{b}}$} & \multirow{2}{*}{ Interpretação } \\
\hline & $\mathbf{N}$ & $\%$ & & & & \\
\hline Centro-Oeste & 4.841 & 10,07 & 8,30 & 0,87 & $-0,10 ; 1,84$ & Estacionária \\
\hline Distrito Federal & 792 & 1,65 & 6,90 & 0,06 & $-1,69 ; 1,85$ & Estacionária \\
\hline Goiás & 2.005 & 4,17 & 8,07 & 0,99 & $-0,29 ; 2,29$ & Estacionária \\
\hline Mato Grosso & 944 & 0,93 & 7,65 & 0,13 & $-1,41 ; 1,70$ & Estacionária \\
\hline Mato Grosso do Sul & 1.101 & 2,29 & 11,40 & 1,94 & 0,$32 ; 3,59$ & Crescente \\
\hline Norte & 4.371 & 9,09 & 6,57 & 2,47 & 1,$93 ; 3,01$ & Crescente \\
\hline Acre & 270 & 0,56 & 9,29 & 5,55 & 2,$40 ; 8,80$ & Crescente \\
\hline Amazonas & 1.229 & 2,56 & 8,12 & 3,81 & 1,$98 ; 5,67$ & Crescente \\
\hline Amapá & 275 & 0,57 & 9,78 & 2,32 & $-0,55 ; 5,27$ & Estacionária \\
\hline Pará & 1.392 & 2,90 & 4,46 & 1,80 & 0,$41 ; 3,20$ & Crescente \\
\hline Rondônia & 513 & 1,07 & 7,63 & 0,98 & $-1,08 ; 3,07$ & Estacionária \\
\hline Roraima & 242 & 0,50 & 13,54 & $-0,69$ & $-2,71 ; 1,36$ & Estacionária \\
\hline Tocantins & 449 & 0,93 & 7,81 & 4,14 & 1,$50 ; 6,84$ & Crescente \\
\hline Nordeste & 11.068 & 23,02 & 5,16 & 2,74 & 1,$51 ; 3,98$ & Crescente \\
\hline Alagoas & 599 & 1,25 & 4,80 & 0,81 & $-0,64 ; 2,29$ & Estacionária \\
\hline Bahia & 1.953 & 4,06 & 3,35 & 3,66 & 1,$72 ; 5,64$ & Crescente \\
\hline Ceará & 2.681 & 5,58 & 8,00 & 1,57 & $-0,47 ; 3,65$ & Estacionária \\
\hline Maranhão & 1.132 & 2,35 & 4,25 & 7,00 & 5,$36 ; 8,66$ & Crescente \\
\hline Piauí & 1.125 & 2,34 & 9,16 & 6,18 & 4,$21 ; 8,19$ & Crescente \\
\hline Pernambuco & 1.735 & 3,61 & 7,33 & 2,23 & $-1,38 ; 5,98$ & Estacionária \\
\hline Paraíba & 639 & 1,33 & 4,26 & 6,78 & 3,$85 ; 9,79$ & Crescente \\
\hline Rio Grande do Norte & 674 & 1,40 & 5,29 & 0,88 & $-0,49 ; 2,27$ & Estacionária \\
\hline Sergipe & 530 & 1,10 & 6,16 & 2,74 & 0,$24 ; 5,30$ & Crescente \\
\hline Sul & 9.635 & 20,04 & 9,18 & $-0,01$ & $-1,30 ; 1,28$ & Estacionária \\
\hline Paraná & 3.451 & 7,18 & 8,40 & 0,35 & $-1,83 ; 2,57$ & Estacionária \\
\hline Rio Grande do Sul & 4.054 & 8,43 & 10,30 & $-1,06$ & $-1,59 ;-0,52$ & Decrescente \\
\hline Santa Catarina & 2.130 & 4,43 & 8,70 & 0,70 & $-0,26 ; 1,67$ & Estacionária \\
\hline Sudeste & 18.161 & 37,78 & 5,79 & 1,00 & 0,$20 ; 1,80$ & Crescente \\
\hline Espírito Santo & 734 & 1,53 & 5,20 & 0,00 & $-1,52 ; 1,55$ & Estacionária \\
\hline Minas Gerais & 5.348 & 11,12 & 6,90 & 2,0 & 0,$95 ; 3,06$ & Crescente \\
\hline Rio de Janeiro & 1.924 & 4,00 & 3,30 & 1,93 & $-0,59 ; 4,51$ & Estacionária \\
\hline São Paulo & 10.155 & 21,12 & 6,20 & 0,38 & $-0,85 ; 1,62$ & Estacionária \\
\hline
\end{tabular}

avariação percentual anual. 'intervalo de confiança da VPA.

Fonte: Sistema de Informação de Mortalidade (SIM), Ministério da Saúde (MS), 1997 a 2019.

Tabela 2. Número, porcentagem de óbitos e tendência da mortalidade proporcional por suicídio segundo local de ocorrência. Brasil, 1997-2019.

\begin{tabular}{lrrrrl}
\hline \multirow{2}{*}{ Local de ocorrência } & \multicolumn{2}{c}{ Óbitos } & \multirow{2}{*}{ VPA $^{\mathbf{a}}$} & \multirow{2}{*}{ IC95\% $^{\mathbf{b}}$} & \multirow{2}{*}{ Interpretação $^{n}$} \\
\cline { 2 - 3 } & $\mathbf{N}$ & \multicolumn{1}{c}{$\%$} & & & \\
\hline Hospital & 9.115 & 18,96 & $-3,94$ & $-4,34 ;-3,53$ & Decrescente \\
Estabelecimento de saúde & 590 & 1,23 & 7,36 & 3,$46 ; 11,40$ & Crescente \\
Domicílio & 26.406 & 54,93 & 1,19 & 0,$87 ; 1,50$ & Crescente \\
Via pública & 3.407 & 7,09 & $-1,00$ & $-2,01 ; 0,02$ & Estacionária \\
Outros & 8.009 & 16,66 & 0,51 & 0,$08 ; 0,94$ & Crescente \\
Ignorados & 549 & 1,14 & $-11,40$ & $-13,52 ;-9,23$ & Decrescente \\
\hline
\end{tabular}

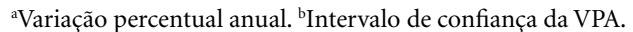


Tabela 3. Número de porcentagem de óbitos e tendência da mortalidade proporcional por suicídio, segundo estado civil. Brasil, 1997-2019.

\begin{tabular}{|c|c|c|c|c|c|}
\hline \multirow{2}{*}{ Estado civil } & \multicolumn{2}{|c|}{ Óbitos } & \multirow{2}{*}{$\mathrm{VPA}^{\mathrm{a}}$} & \multirow{2}{*}{ IC95\% ${ }^{\mathrm{b}}$} & \multirow{2}{*}{ Interpretaçãc } \\
\hline & $\mathbf{N}$ & $\%$ & & & \\
\hline Solteiro & 37.988 & 79,02 & 0,05 & $-0,33 ; 0,43$ & Estacionária \\
\hline Casado & 4.558 & 9,48 & $-4,49$ & $-5,13 ;-3,85$ & Decrescente \\
\hline Viúvo & 88 & 0,18 & $-0,43$ & $-2,96 ; 2,17$ & Estacionária \\
\hline Separado & 546 & 1,14 & 0,63 & $-1,04 ; 2,32$ & Estacionária \\
\hline Outro & 1.837 & 3,82 & 4,60 & $-5,50 ; 15,78$ & Estacionária \\
\hline Ignorado & 3.059 & 6,36 & 0,65 & $-0,32 ; 1,63$ & Estacionária \\
\hline
\end{tabular}

aariação percentual anual. ' Intervalo de confiança da VAP.

Fonte: Sistema de Informação de Mortalidade (SIM), Ministério da Saúde (MS), 1997 a 2019.

que possuem capacidade insuficiente dos sistemas de registros e, ainda, por ser o suicídio um assunto cercado de estigma e tabu ${ }^{2}$. Outro aspecto refere-se à dificuldade em registrar a causa da morte, como por exemplo, o acidente automobilístico, afogamento e morte por causa indefinida, e esses contribuem para ocultar a dimensão real do problema ${ }^{6,21}$.

Entre os sexos, os maiores coeficientes de mortalidade por suicídio entre os homens comparados às mulheres, corroboram com os achados mundiais ${ }^{2,17}$. Outros países como o Equador ${ }^{18}$ e Portugal ${ }^{17}$, por exemplo, também evidenciam a maior ocorrência do fenômeno entre os homens. Contudo, embora os homens sejam mais propensos a cometer o suicídio, constatou-se por meio deste estudo que ambos os sexos apresentaram aumento das taxas de suicídio.

As disparidades nas taxas de suicídio entre os sexos podem ser atribuídas aos diferentes papéis sociais que cada indivíduo desempenha, uma vez que o homem é visto como o mantenedor econômico e possui estressores relacionados às questões do trabalho e de provimento financeiro. As mulheres estão menos expostas a esses fatores, que são considerados de risco para o suicídio, no entanto, enfrentam problemas relacionados a aspectos de saúde, familiares e histórico de abuso físico e sexual, o que também podem ser determinantes para o ato suicida ${ }^{17,21,22}$. Enfatiza-se ainda que os homens se preocupam menos com sua saúde, principalmente a saúde mental ${ }^{23}$ e por consequência, procuram com menos frequência os serviços de saúde.

Outro fator importante quando se discute a diferença do ato suicida entre homens e mulheres refere-se ao método utilizado, sendo que os homens comumente apresentam características mais impulsivas, optam por meios considerados mais letais, como o enforcamento e armas de fogo, enquanto as mulheres utilizam meios que podem ser reversíveis, como a intoxicação medicamentosa $^{12,17}$.

As lesões autoprovocadas (X70-X84), apresentaram maior proporção quando comparadas com a autointoxicação (X60-X69) nesta pesquisa. Outros estudos desenvolvidos no Brasil também identificaram que as mortes por suicídio ocorrem maiormente por lesões autoprovocadas $^{21,24}$. A lesão autoprovocada é a violência que a pessoa inflige a si mesmo, seja por arma de fogo, por arma branca e objetos contundentes, por enforcamento e estrangulamento, por precipitação de lugar elevado ou outros ${ }^{25}$, meios mais letais e mais comumente utilizados pelos homens ${ }^{12}$.

É fato que a acessibilidade aos meios pode ser determinante para o desfecho do fenômeno ${ }^{12,26}$. Um estudo realizado em Portugal que objetivou identificar o padrão de tendência do suicídio registrado no país entre os anos de 2007 e 2014, evidenciou um crescimento de mortes por armas de fogo, e, uma hipótese para esse achado refere-se ao acesso facilitado por meio de vendas de armas ilegais ${ }^{17}$. Uma revisão de literatura demonstrou que o acesso a arma de fogo é considerado um preditor para o elevado índice de suicídio entre policiais e profissionais das forças armadas ${ }^{27}$. A grande quantidade de armas de fogo ilegais em circulação e o fácil acesso a esse meio é um fator de risco para o indivíduo, pois ao se deparar com um problema a arma será vista como um meio de solução, o que pode ocasionar no aumento do suicídio $^{28}$.

Visando a prevenção do suicídio o governo da Austrália reduziu o número de acesso a armas de fogo, por meio de campanhas e medidas de controle de compra e porte de arma, contribuindo significativamente para sua queda na popu- 
lação geral $^{29}$. Uma revisão sistemática apontou evidências para a prevenção do suicídio, como a restrição do acesso a meios letais, especialmente no que diz respeito ao controle de analgésicos e pontos de acesso para suicídio por precipitação de lugar elevado. Tratamentos farmacológicos e psicológicos para depressão também são importantes nesta prevenção ${ }^{30}$.

Com relação às regiões do país, a Sul apresentou o maior coeficiente de mortalidade por suicídio assim como descrito por outras pesquisas brasileiras ${ }^{6,21}$, mantendo tendência estável ao longo dos anos. Contudo, estudo anterior desenvolvido com a população brasileira identificou uma tendência decrescente na região Sul, sendo que a estabilização/redução pode ocorrer devido a melhoria das notificações, assim como investimentos em ações de sensibilização dos profissionais para a importância do efetivo preenchimento das fichas ${ }^{6}$. Ainda nessa região, observa-se que o Rio Grande do Sul foi o único entre as UF que apresentou tendência decrescente. Tal fato pode ter ocorrido em decorrência do reflexo das ações desenvolvidas pelo "Programa de Prevenção ao Suicídio" (PPS), criado com a finalidade de estabelecer uma linha de cuidado e capacitação profissional na identificação de riscos para o suicídio $^{6}$, o que poderia ser utilizado como experiência exitosa no incentivo a tal estratégia para os demais estados brasileiros.

A tendência do suicídio em adultos jovens mostrou-se crescente nas regiões Nordeste, Norte e Sudeste, sendo que, entre as 11 UF que apresentaram crescimento, nove são do Norte e Nordeste. As características do território relacionado às condições de vida como uma maior exposição a situações de vulnerabilidade que envolve a desigualdade social, falta de acesso à saúde, a baixa escolaridade, a exposição à violência e outros fatores, podem produzir impactos negativos na saúde do indivíduo favorecendo o desencadeamento de sofrimento psíquico e levá-lo ao suicídio $^{21}$. Portanto, apesar das diversidades culturais, socioeconômica e demográficas de cada região, é fundamental o desenvolvimento de estratégias que visem a prevenção e a redução da ocorrência de casos de suicídio nos diferentes contextos.

Assim como neste estudo, outro realizado no México que buscou apresentar a tendência de suicídio no período de 1990 a 2011 na população geral, identificou maior ocorrência do ato suicida nos domicílios, por ser um local com mais facilidade em consumá-lo, além de representar um espaço com carga emocional, gerando maior impacto sobre os membros familiares ${ }^{31}$. Em re- lação aos estabelecimentos de saúde, diversos fatores contribuem para a ocorrência do fenômeno, sendo eles o despreparo da equipe, a falta de infraestrutura das instituições, tais como janelas sem proteção, facilidade aos meios dispostos, medicamentos, instrumentos perfurocortantes, assim como situações emocionais relacionadas à internação prolongada e agravamento da condição clínica, podem desencadear a ansiedade e depressão que são fatores que aumentam o risco para o suicídio ${ }^{10}$.

Os solteiros obtiveram a maior proporção nos casos de suicídio, assim como em outros estudos $^{32,33}$. Uma hipótese para tal achado seria devido a maior exposição ao isolamento social por parte dos solteiros, sendo essa uma característica considerada um fator predisponente para o comportamento suicida ${ }^{10}$. Por sua vez, os casados expressaram declínio na taxa de mortalidade por suicídio. A consolidação dos vínculos sociais, bem como da rede familiar têm sido amplamente descritos na literatura como fatores de proteção ao suicídio ${ }^{31}$.

Embora apresente limitações próprias do tipo de estudo, os resultados obtidos são inéditos e relevantes para a compressão do fenômeno, pois trazem a tendência de suicídio em adultos jovens brasileiros, em uma análise de 23 anos. Nesse sentido, os achados do estudo demonstram a relevância da temática enquanto um problema de saúde pública e permitem a compreensão do comportamento suicida nessa faixa etária, necessária para a elaboração de políticas de saúde nacionais e regionais, que visem programas de promoção da saúde mental, prevenção ao suicídio, tratamento oportuno de transtorno mental e/ou incapacidades decorrentes de tentativas anteriores.

\section{Conclusão}

Conclui-se que a tendência das taxas de mortalidade por suicídio em adultos jovens de 20 a 29 anos no Brasil entre 1997 e 2019 foi crescente nessa faixa etária e em ambos os sexos. Houve tendência de aumento do suicídio nas regiões Norte, Nordeste e Sudeste e que ocorreram no âmbito domiciliar, em outros estabelecimentos de saúde e em outros locais.

O estudo permitiu traçar o diagnóstico situacional do fenômeno no país, podendo servir como retrato atual para subsidiar a formulação de políticas por órgãos privados e governamentais. Além disso, faz-se necessário que os pro- 
fissionais de saúde atuem de forma interprofissional, interdisciplinar e intersetorial frente ao evento, sobretudo na implementação de práticas baseadas em evidências.

\section{Colaboradores}

VL Arruda contribuiu para o delineamento do estudo, da coleta e análise dos dados, da interpretação dos dados e da redação do artigo. BHBM Freitas e SR Marcon contribuíram para a concepção e do delineamento do estudo e da revisão crítica do artigo. FY Fernandes e NVP Lima colaboraram com a coleta de dados e da revisão crítica do artigo. J Bortolini participou do delineamento do estudo e da revisão crítica do artigo. Todos os autores aprovaram a versão a ser publicada. 


\section{Referências}

1. Vieira RG, Almeida CFR, Rodrigues G, Gonçalves SS, França AS, Oliveira MB. Prevalência e risco de suicídio no Brasil e na cidade de Barra do Garças (MT): revisão de literatura. Rev Debate Psiquiatr 2017; 19(3):445-453.

2. World Health Organization (WHO). Suicide data. Geneva: WHO; 2018.

3. Minayo MCS, Pinto LW, Assis SG, Cavalcante FG, Mangas RMN. Trends in suicide mortality among Brazilian adults and elderly, 1980-2006. Rev Saude Publica 2012; 46(2):300-309.

4. Geulayov G, Casey D, McDonald KC, Foster P, Pritchard K, Wells C, Clements C, Kapur N, Ness J, Waters $\mathrm{K}$, Hawton K. Incidence of suicide, hospital-presenting non-fatal self-harm, and community-occurring non-fatal self-harm in adolescents in England (the iceberg model of self-harm): a retrospective study. Lancet Psychiatry 2018; 5(2):167-174.

5. Alicandro G, Malvezzi M, Gallus S, La Vecchia C, Negri E, Bertuccio P. Worldwide trends in suicide mortality from 1990 to 2015 with a focus on the global recession time frame. Int J Public Health 2019; 64(5):785-795.

6. D'Eça Júnior A, Rodrigues LS, Meneses Filho EP, Costa LDLN, Rêgo AS, Costa LC, Batista RFL. Mortalidade por suicídio na população brasileira, 1996-2015: qual é a tendência predominante? Cad Saude Coletiva 2019; 27(1):20-24.

7. Figueiredo AEB. Suicida: avaliação e manejo. Cien Saude Colet 2016; 21(11):3633-3634.

8. Sinyor M, Tse R, Pirkis J. Global trends in suicide epidemiology. Curr Opin Psychiatry 2017; 30(1):1-6.

9. Bahia CA, Avanci JQ, Pinto LW, Minayo MCS. Lesão autoprovocada em todos os ciclos da vida: Perfil das vítimas em serviços de urgência e emergência de capitais do Brasil. Cien Saude Colet 2017; 22(9):2841-2850.

10. Botega NJ. Comportamento suicida: Epidemiologia. Psicol USP 2014; 25(3):231-236.

11. Melo CDF, Sousa JC, Martins SM, Frota PC. Brazilian population perception about suicide. Rev Pesqui Cuid Fundam Online 2018; 10(4):1085.

12. Ribeiro NM, Castro SS, Scatena LM, Haas VJ. Time-trend analysis of suicide and of health information systems in relation to suicide attempts. Texto Context Enferm 2018; 27(2):1-11.

13. Antunes JLF, Cardoso MRA. Uso da análise de séries temporais em estudos epidemiológicos. Epidemiol Serv Saude 2015; 24(3):565-576.

14. Brasil. Ministério da Saúde (MS). Perfil epidemiológico das tentativas e óbitos por suicídio no Brasil e a rede de atenção à saúde. Bol Epidemiol 2017; 48(30):114.

15. Kino S, Jang SN, Gero K, Kato S, Kawachi I. Age, period, cohort trends of suicide in Japan and Korea (1986-2015): A tale of two countries. Soc Sci Med 2019; 235:112385.

16. Spiller HA, Ackerman JP, Spiller NE, Casavant MJ. Sex- and Age-specific Increases in Suicide Attempts by Self-Poisoning in the United States among Youth and Young Adults from 2000 to 2018. J Pediatr 2019; 210:201-208
17. Nunes AM. Suicide in Portugal: Image of the country. J Bras Psiquiatr 2018; 67(1):25-33.

18. Gerstner RM, Lara Lara F. Análisis de tendencias temporales del suicidio en niños, adolescentes y adultos jóvenes en Ecuador entre 1990 y 2017. An Sist Sanit Navar 2019; 42(2010):9-18.

19. Ribeiro JM, Moreira MR. An approach to suicide among adolescents and youth in Brazil. Cien Saude Colet 2018; 23(9):2821-2834.

20. Sobrinho AT, Campos RC. Perceção de acontecimentos de vida negativos, depressão e risco de suicídio em jovens adultos. Anal Psicol 2016; 34(1):47-60.

21. Machado DB, Santos DN. Suicídio no Brasil, de 2000 a 2012. J Bras Psiquiatr 2015; 64(1):45-54.

22. Stone DM, Holland KM, Schiff LB, McIntosh WLKW. Mixed Methods Analysis of Sex Differences in Life Stressors of Middle-Aged Suicides. Am J Prev Med 2016; 51(5):S209-S218.

23. Soares RJO, Nascimento FPB. Suicídio e Tentativa de Suicídio: Contribuições da Enfermagem Brasileira. $J$ Health Sci 2017; 1(19):19-24.

24. Fernandes FY, Freitas BHBM, Marcon SR, Arruda VL, Lima NVP, Bortolini J, Gaíva MAM. Tendência de suicídio em adolescentes brasileiros entre 1997 e 2016. Epidemiol Serv Saude 2020; 29(4):e2020117.

25. Monteiro RA, Bahia CA, Paiva EA, Sá NNB, Minayo MCS. Hospitalizations due to self-inflicted injuries - Brazil, 2002 to 2013. Cien Saude Colet 2015; 20(3):689-700.

26. Santos EGO, Oliveira YOMC, Azevedo UN, Nunes ADS, Amador AE, Barbosa IR. Spatial temporal analysis of mortality by suicide among the elderly in Brazil. Rev Bras Geriatr Gerontol 2017; 20(6):845-855.

27. Franco FM. Fatores de risco, fatores protetivos e prevenção do suicídio entre policiais e outros agentes da lei : perspectiva internacional. Rev Psicol Saude Ment Segurança Publica 2018; 7:97-114.

28. Almeida LB, Neres WFS. A fragiliade e ineficácia do estatuto do desarmamento frente ao combate de armas de fogo ilegais no estado de Goiás [Internet]. Comando da Academia da Polícia Militar de Goiás. Programa de Estudos Pós-Graduação da PMGO Turma Golf.; 2018 [acessado 2019 dez 10]. Disponível em: https://acervodigital.ssp.go.gov.br/pmgo/bitstream/123456789/921/3/Lucas Bueno D Almeida. pdf.

29. Martin G. On guns and suicide. Adv Ment Heal 2013; 11(2):118-121.

30. Zalsman G, Hawton K, Wasserman D, van Heeringen K, Arensman E, Sarchiapone M, Carli V, Höschl C, Barzilay R, Balazs J, Purebl G, Kahn JP, Sáiz PA, Lipsicas CB, Bobes J, Cozman D, Hegerl U, Zohar J. Suicide prevention strategies revisited: 10-year systematic review. Lancet Psychiatry 2016; 3(7):646-659.

31. Jiménez-Ornelas RA, Cardiel-Téllez L. El suicidio y su tendencia social en México: 1990-2011. Papeles Poblac 2013; 19(77):205-229.

32. Oliveira LR, Benedetti ADOC. Suicídio em Mato Grosso - Brasil: 1996 a 2015. J Heal Biol Sci 2018; 6(4):391. 
33. Vidal CEL, Gontijo ECDM, Lima LA. Tentativas de suicidio: Factores pronósticos y estimativa del exceso de mortalidad. Cad Saude Publica 2013; 29(1):175187.

Artigo apresentado em 30/01/2020

Aprovado em 14/04/2021

Versão final apresentada em 16/04/2021

Editores-chefes: Romeu Gomes, Antônio Augusto Moura da Silva 